\title{
La educación como fundamento orientador hacia una cultura ambiental
}

\author{
Education as a Guiding Foundation Towards an Environmental Culture
}

\section{A educação como base norteadora de uma cultura ambiental}

\author{
Ma. de los Ángeles Martínez Ortega \\ Instituto Politécnico Nacional, México \\ mmartinezo@ipn.mx \\ https://orcid.org/0000-0002-8483-9844
}

\section{Resumen}

La presente investigación surge de la premisa de que la mujer como madre es considerablemente más sensible hacia el cuidado del medio ambiente, es la que inicialmente tiene un vínculo directo con el individuo y, por lo tanto, es necesario reeducarla para que sea la guiadora inicial hacia la construcción de una cultura ambiental. El objetivo principal fue señalar la importancia de la educación dirigida hacia la conformación de una cultura de este tipo, partiendo de un enfoque de género y su implicación y dirección hacia la generación de una sensibilidad ambiental, en donde se exalte que el acontecer del ser humano impacta directamente en el medio ambiente y, por extensión, repercute en el cambio climático. Se partió de dos preguntas eje: ¿el género y el nivel académico influyen de manera directa en la respuesta sensible del individuo hacia el cuidado del medio ambiente?, ¿la incorporación más directa de la mujer propiciará una educación ambiental que repercutirá en la construcción de una cultura ambiental? La investigación tuvo un enfoque mixto (cuantitativo y cualitativo); se trató de un estudio transversal que incluyó una encuesta semiestructurada. Inicialmente se determinaron dos variables cualitativas nominales-ordinales: Género (dicotómica), variable independiente, y Sensibilidad Ambiental (politómica), esto es, la 


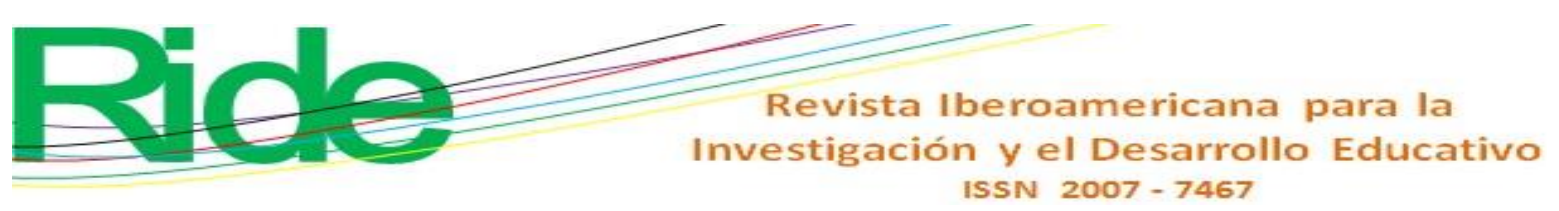

conciencia del impacto negativo del comportamiento humano hacia el medio ambiente, como variable dependiente. Posteriormente, por necesidades del estudio, se incluyó la variable Nivel Académico (semestre escolar) (politómica). El análisis se realizó a través del coeficiente ji al cuadrado, utilizando el software IBM SPSS Statistics 23 para medir la relación de las variables. Los resultados arrojaron, en primera instancia, que no existe relación directa entre Género y Sensibilidad Ambiental (conciencia del impacto negativo de nuestras actividades cotidianas hacia el medio ambiente del individuo). Sin embargo, con la incorporación de la variable Nivel Académico (semestre), se comprobó que sí existe correlación directa entre las variables, esto es, el género femenino manifestó tener una mayor sensibilidad del impacto que generan sus acciones cotidianas en el medio ambiente. Se concluye que se deben promover acciones que involucren de manera directa a la mujer hacia una reeducación ambiental, en donde se le permita actuar de manera directa en esta acción proactiva y axiológica. Por lo tanto, una propuesta directa es trabajar hacia una educación ambiental, ya que es un factor importante para tomar conciencia del trabajo en pro de un desarrollo sostenible desde casa, completarlo en la escuela y vivenciarlo en la sociedad, todo ello hacia una cultura ambiental.

Palabras clave: cultura ambiental, educación ambiental, género, nivel académico, sensibilidad ambiental.

\section{Abstract}

The present investigation arises from the premise that the woman as mother is considerably more sensitive towards caring for the environment, it is the one that initially has a direct link with the individual and, therefore, it is necessary to re-educate her to be the initial guide towards construction of an environmental culture. The main objective was to point out the importance of education directed towards the conformation of an environmental culture, starting from a gender perspective and its implication and direction towards the generation of an environmental sensitivity, where it is exalted that the happening of the human being directly impacts in the environment and therefore has an impact on climate change. Two main questions were started: Does gender and academic level directly influence the sensitive response of the individual towards caring for the environment? Will the more direct incorporation of women promote environmental education, which will have an impact on building an environmental culture? The research was with a mixed approach (quantitative and qualitative); a cross-sectional study with a semi-structured 


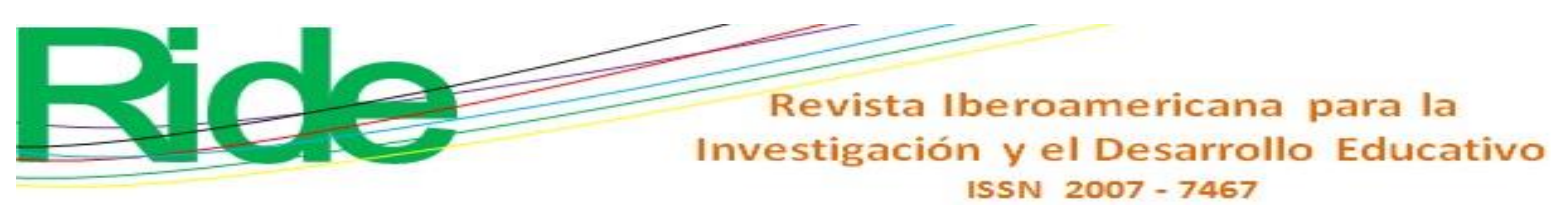

survey. The study began with the analysis of two nominal-ordinal qualitative variables, Gender (dichotomous) as an independent variable and Environmental Sensitivity, as awareness of the negative impact of human behavior towards the environment (polytomous), as a dependent variable. Additionally, due to study needs, the variable Academic Level (school semester) (polytomic) was subsequently included. The analysis was performed through the chi-square coefficient, using the IBM SPSS Statistics 23 software to measure the relationship of the variables. The results showed, in the first instance, that there is no direct relationship between Gender and Environmental Sensitivity (awareness of the negative impact of our daily activities towards the environment of the individual). Given this situation, the variable Academic Level (semester) was subsequently incorporated. With this adaptation, it was verified that there is a direct correlation of the variables, that is, the feminine gender manifested having a greater sensitivity of the impact that their daily actions generate to the environment. It was concluded that actions that directly involve women towards environmental reeducation should be promoted, where they are allowed to act directly in this proactive and axiological action. Therefore, a direct proposal is to work towards an environmental education, since it is an important factor to become aware of the work in favor of sustainable development from home, complete it at school and experience it in society, all towards an environmental culture.

Keywords: environmental culture, environmental education, gender, academic level, environmental sensitivity.

\section{Resumo}

A presente investigação parte da premissa de que a mulher como mãe é consideravelmente mais sensível ao cuidado com o meio ambiente, é ela quem inicialmente tem um vínculo direto com o indivíduo e, portanto, é necessário reeducá-la para ser o guia inicial. para a construção de uma cultura ambiental. O principal objetivo foi apontar a importância da educação voltada para a conformação de uma cultura desse tipo, com base na abordagem de gênero e suas implicações e direcionamento para a geração de uma sensibilidade ambiental, onde se destaca que os eventos do ser humano afeta diretamente o meio ambiente e, por extensão, afeta as mudanças climáticas. $O$ ponto de partida foram duas questões centrais: gênero e nível acadêmico influenciam diretamente a resposta sensível do indivíduo em relação ao cuidado com o meio ambiente? A incorporação mais direta de mulheres promoverá educação ambiental que terá impacto na construção de uma 


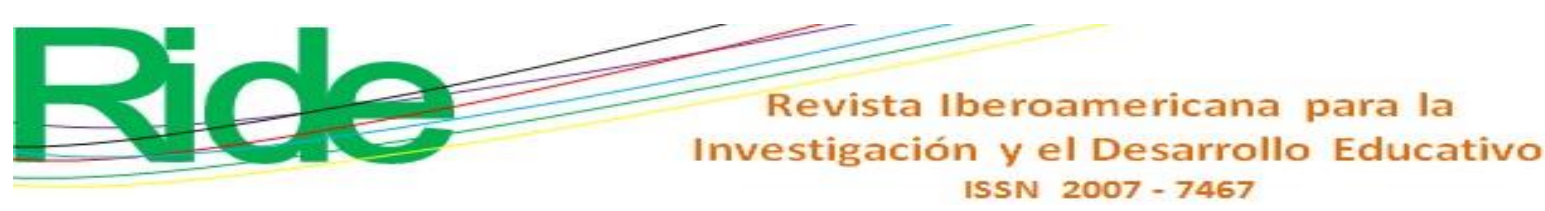

cultura ambiental? A pesquisa teve uma abordagem mista (quantitativa e qualitativa); foi um estudo transversal que incluiu uma pesquisa semiestruturada. Inicialmente, foram determinadas duas variáveis qualitativas nominais-ordinais: gênero (dicotômico), variável independente e sensibilidade ambiental (politômico), ou seja, conscientização do impacto negativo do comportamento humano sobre o meio ambiente, como variável dependente. Posteriormente, devido às necessidades do estudo, foi incluída a variável Nível Acadêmico (semestre letivo) (politômica). A análise foi realizada através do coeficiente de qui quadrado, utilizando o software IBM SPSS Statistics 23 para medir a relação das variáveis. Os resultados mostraram, em primeira instância, que não há relação direta entre gênero e sensibilidade ambiental (conscientização do impacto negativo de nossas atividades diárias no ambiente do indivíduo). Porém, com a incorporação da variável Nível Acadêmico (semestre), verificou-se uma correlação direta entre as variáveis, ou seja, o sexo feminino se manifestou com maior sensibilidade ao impacto gerado por suas ações cotidianas no meio ambiente. Conclui-se que ações que envolvam diretamente as mulheres na reeducação ambiental devem ser promovidas, onde elas podem atuar diretamente nessa ação pró-ativa e axiológica. Portanto, uma proposta direta é trabalhar em prol da educação ambiental, pois é um fator importante conhecer o trabalho a favor do desenvolvimento sustentável em casa, concluí-lo na escola e vivenciá-lo na sociedade, tudo em prol de uma cultura ambiental. Palavras-chave: cultura ambiental, educação ambiental, gênero, nível acadêmico, sensibilidade ambiental.

Fecha Recepción: Diciembre 2019

Fecha Aceptación: Abril 2020 


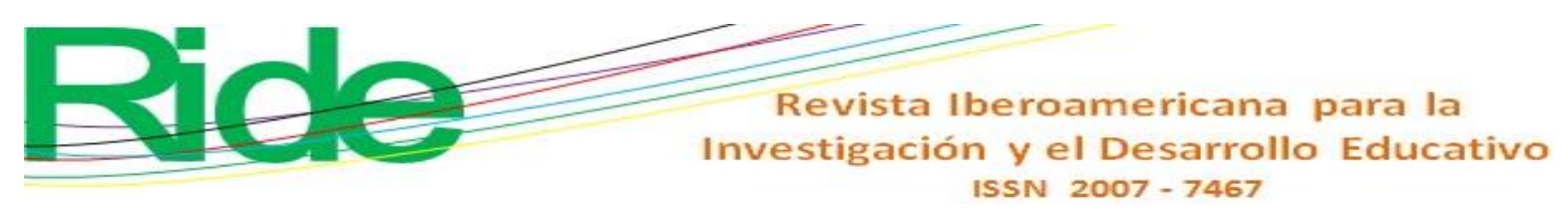

\section{Introducción}

Actualmente, a través de la adopción de la Agenda 2030 (Organización de las Naciones Unidas para la Educación, la Ciencia y la Cultura [Unesco], 2018), la cual forma parte de un movimiento global para erradicar la pobreza a través de 17 objetivos de desarrollo sostenible para el 2030, la educación ha adquirido un nuevo ímpetu. Específicamente, el objetivo cuatro de esta agenda apunta a la universalización de una educación incluyente y permanente (Unesco, 2018).

Para la Unesco (2018), la educación es considerada como un derecho humano básico y, además, la base sobre la cual construir la paz e impulsar el desarrollo sostenible. La educación para el desarrollo sostenible se entiende como una que fomenta los cambios en el conocimiento, las habilidades, los valores y las actitudes para permitir una sociedad más sostenible y justa para todos. Tiene como objetivo empoderar y equipar a las generaciones actuales y futuras para satisfacer sus necesidades utilizando un enfoque equilibrado e integrado: toma en consideración las dimensiones económica, social y ambiental del desarrollo sostenible (Unesco, 2018). Un ejemplo de ello es la red de voluntarios denominada Guardarenas, la cual tiene como finalidad cambiar el estilo de vida de niños, jóvenes y adultos a escala comunitaria, creando conciencia sobre la fragilidad de los entornos marinos y costeros y la necesidad de utilizarlos con prudencia. Esta red pone en acción fundamento científicos de manera interdisciplinaria, con usos que van desde la geografía hasta el arte, desde la poesía hasta las matemáticas (Unesco, 2010).

Así pues, ante los acontecimientos de desastres naturales, causados por el deterioro ambiental y cambio climático, se presume que la educación para el desarrollo sostenible no es una opción sino una prioridad (Unesco, 2005, 2006a). En esa línea, el desarrollo sostenible involucra una visión diferente, promoviendo una actitud lo suficientemente proactiva para visualizar una calidad de vida para todos, tanto de las presentes como de las futuras generaciones, buscando un equilibrio entre el crecimiento económico, social y la protección del medio ambiente (Unesco, 2005, 2016a). La sostenibilidad, al igual que la resiliencia, se puede aplicar en cualquier ámbito, presupone una respuesta incluyente que involucra la capacidad de identificar las fortalezas y debilidades, manifestar una respuesta de adaptación, ser disruptivos y enfrentar los retos, en especial maximizar los recursos disponibles para asegurar una larga y, desde luego, productiva vida en beneficio de los interesados, todo ello en sociedad (KPMG en México, 2018).

Actualmente en México se tiene la meta de comenzar a mejorar las condiciones materiales de las escuelas del país, y con ello garantizar el acceso de todos los jóvenes a la educación (Presidencia de la República, 2019). Esto debido, en parte, a que el rezago porcentual de jóvenes 


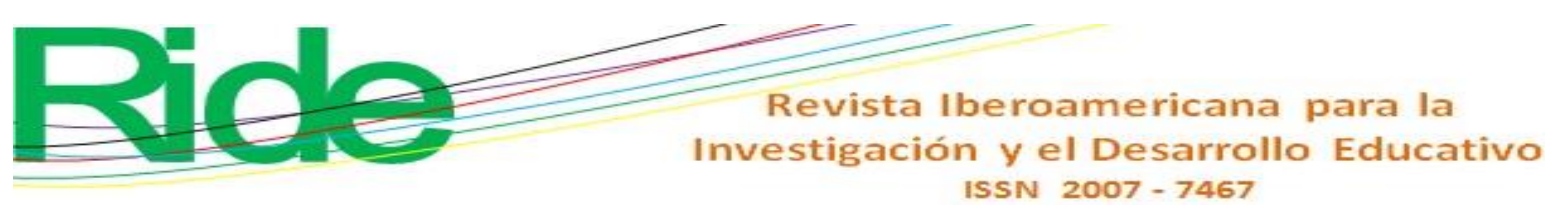

mexicanos de 25 a 34 años con estudios superiores está por debajo del promedio de la Organización para la Cooperación y el Desarrollo Económicos [OCDE] (2019) (44 \%), inclusive a pesar de incrementarse de 16 \% a 23 \% durante el periodo 2007-2017. En 2018, la Secretaría de Educación Pública (SEP) invitó a la OCDE a realizar una revisión de esta condición. La pretensión es que emprenda un nuevo estudio para examinar las fortalezas y debilidades de la gobernanza y de las estrategias que guían este sistema, así como del análisis de mecanismos externos de aseguramiento de la calidad, las condiciones para la equidad, entre otros aspectos (OCDE, 2019).

\section{Vamos hacia una educación ambiental}

Es pertinente mencionar que la Unesco, perteneciente a la Organización de las Naciones Unidas (ONU), es la única revestida con el privilegio de involucrarse en todos los aspectos referentes a la educación. En esa línea, en el documento Sustainable Development Data Digest: Laying the Foundation to Measure Sustainable Development Goal 4, la Unesco (2016b) especifica estrategias puntuales y actores fundamentales, involucrados directamente para promover un desarrollo sostenible desde la educación.

Teniendo en cuenta lo anterior, las preguntas complementarias que guiaron esta investigación fueron las siguientes: ¿qué tan familiarizados están los alumnos hacia una educación ambiental?, ¿cómo influyen los elementos que involucran una educación ambiental en su devenir?, ¿cuál es el efecto que tiene en los alumnos el ser proactivos en su devenir académico?, ¿cuál es el conocimiento que tienen los alumnos sobre información ambiental?, ¿cuántos alumnos tienen conocimiento de su entorno ambiental?, ¿cómo impacta en su actuar cotidiano esta información?, ¿cuál es la manifestación referente a la sensibilidad ambiental?, ¿cuántos alumnos son conscientes del impacto al medio ambiente por sus actividades cotidianas?, ¿cuál es la sensibilización por género del impacto ambiental?, ¿cuál es el efecto académico sobre la responsabilidad de cuidar el medio ambiente, siendo conscientes del impacto del acontecer cotidiano?

Finalmente, se definieron de las siguientes preguntas de investigación: ¿el género y el nivel académico influyen de manera directa en la respuesta sensible del individuo hacia el cuidado del medio ambiente?, ¿la incorporación más directa de la mujer propiciará una educación ambiental que repercutirá en la construcción de una cultura ambiental? 


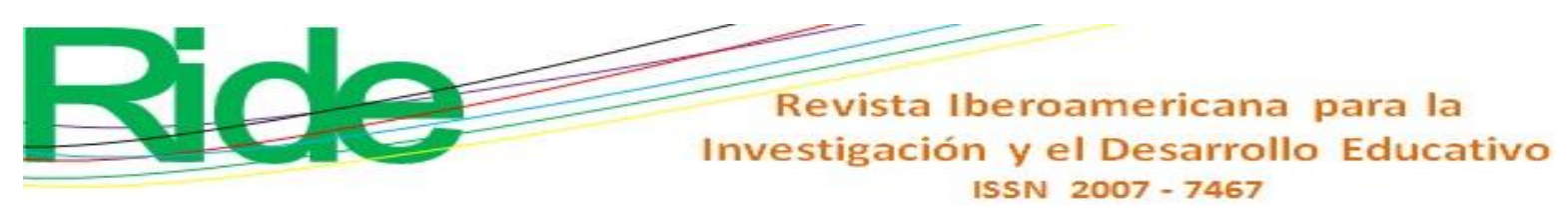

\section{Educación ambiental}

Cada país tiene su propio conjunto de leyes que ayuda a controlar las emisiones de gases contaminantes y disminuir el impacto negativo en el medio ambiente por parte de las industrias y la sociedad. Estas leyes y normas van acompañadas por multas o sanciones que se deberán seguir al pie de la letra para poder garantizar un verdadero cambio en la sociedad. Sin embargo, para alcanzar el objetivo planteado, se requiere un cambio radical en el ser humano: prescindir del enfoque punitivo y dirigirse hacia una cultura ambiental. De acuerdo con Miranda (2013):

La cultura ambiental es la forma como los seres humanos se relacionan con el medio ambiente, y para comprenderla se debe comenzar por el estudio de los valores; estos, a su vez, determinan las creencias y las actitudes y, finalmente, todos son elementos que dan sentido al comportamiento ambiental (p. 94).

Por otro lado, en cuanto a la cuestión de género, se partió de la definición que maneja la investigadora Leticia Santín del Río (2011), quien manifiesta, de manera similar a lo arriba mencionado, que "la mejor forma de la paridad de género es la que no tiene que ser promulgada ni declarada como algo obligatorio, sino que es el resultado de un avance social” (p. 305). Es de vital importancia que se considere el papel tan importante que tiene la mujer en el impacto ambiental, ya que, por naturaleza, está inclinada a la preservación de la vida. Por lo tanto, en esta búsqueda de equidad de género se debe de ver a la mujer no como víctima, sino como actor central para avanzar hacia la sostenibilidad. No obstante, a nivel internacional, se perfilan consecuencias negativas por las diferencias del género. En el documento Global Gender and Environment Outlook del Programa de las Naciones Unidas para el Medio Ambiente [UNEP] (2016), desarrollado por un equipo de casi 50 expertos, queda claro que la desigualdad de género es una de las amenazas más generalizadas para el desarrollo sostenible. La desigualdad de género tiene impactos negativos en el acceso, uso y control de una amplia gama de recursos, y en la capacidad de cumplir con las obligaciones de derechos humanos con respecto al disfrute, por parte de mujeres y hombres, de un ambiente limpio, seguro, saludable y sostenible (UNEP, 2016).

A nivel Latinoamérica, a finales de los 90, en Chile se presentó un trabajo en donde se manifestó la necesidad del conocimiento sobre las formas en que las mujeres de diferentes grupos y sectores de la sociedad han participado en el desarrollo, y se puso en relieve la interconexión entre género, medio ambiente y sostenibilidad (Rico, 1998). 


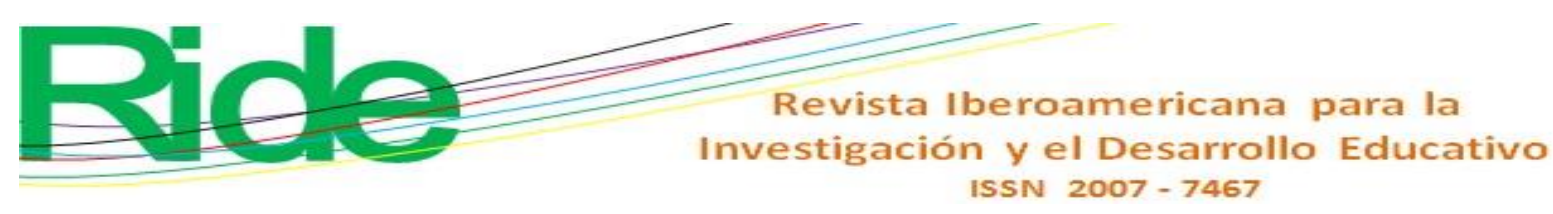

del cuidado del medio ambiente. Se validó el instrumento de medición con el coeficiente de Cronbach; el resultado fue de 0.79 .

Siguiendo a Hernández, Fernández y Baptista (2004), una vez realizada la validación del instrumento, se aplicó a una muestra probabilística-estratificada de 173 elementos de una población de 690 estudiantes en el 2017 en la ESIA Unidad Tecamachalco del IPN. La investigación en dicha unidad educativa se aplicó únicamente a la comunidad estudiantil.

Para la fundamentación de los resultados duros, se realizó el análisis estadístico a través de la prueba de ji al cuadrado, utilizando el paquete de software IBM SPSS Statistics 23 (paquete estadístico para las ciencias sociales) para analizar la relación de las variables. Primeramente, se consideraron dos: Género como variable independiente y Sensibilidad Climática (el impacto negativo de sus actividades cotidianas en el deterioro ambiental) como variable dependiente. Posteriormente, se incluyó la variable Nivel Académico (semestre escolar), también independiente.

Se partió de la inferencia de que el género y el nivel académico impactan directamente en la respuesta del individuo hacia el cuidado del medio ambiente. Así, se planteó la H0 (hipótesis nula), en donde se manifiesta que estas variables no están relacionadas.

Posteriormente, por los resultados observados en un inicio (y que manifestaban no tener relación), se incluyó una tercera variable, Nivel Académico, como ya se mencionaba líneas arriba. A continuación, en la figura 1, se describe el modelo propuesto.

Figura 1. Variables del modelo

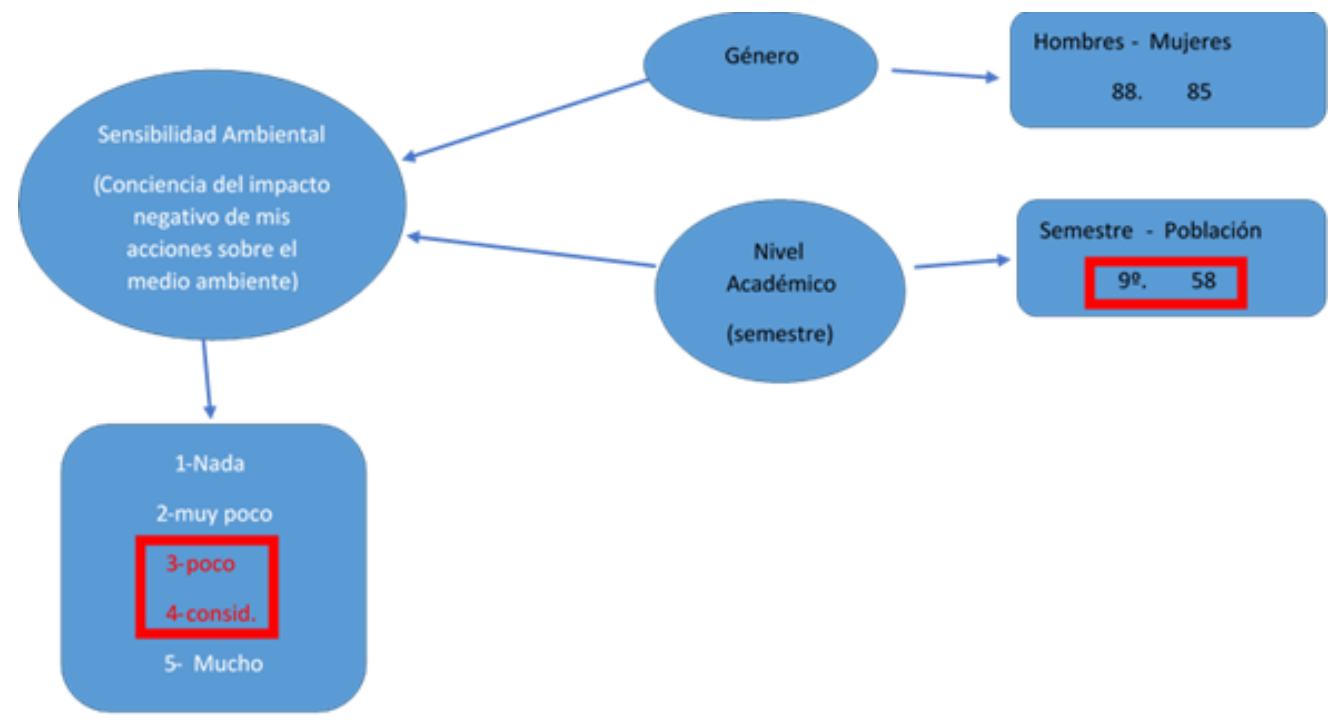

Fuente: Elaboración propia 


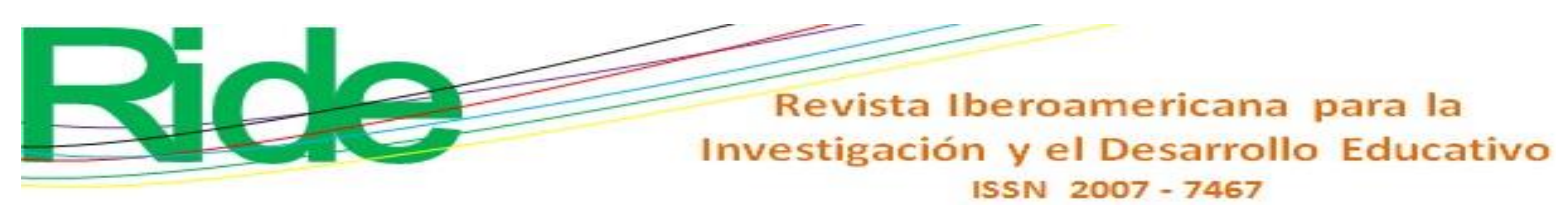

Tabla 1. Análisis de variables

\begin{tabular}{|c|c|c|c|c|}
\hline Variables & Conceptualización & Dimensión & Indicadores & $\begin{array}{c}\text { Parámetro de } \\
\text { medición }\end{array}$ \\
\hline $\begin{array}{l}\text { Sensibilidad } \\
\text { Ambiental }\end{array}$ & $\begin{array}{l}\text { Variable } \\
\text { dependiente } \\
\text { Vinculación del } \\
\text { término en su } \\
\text { devenir y conciencia } \\
\text { del impacto negativo } \\
\text { de su acontecer en el } \\
\text { medio ambiente. }\end{array}$ & $\begin{array}{l}\text { ESIA Unidad } \\
\text { Tecamachalco } \\
\text { del IPN }\end{array}$ & $\begin{array}{l}\text { Nivel del } \\
\text { impacto negativo } \\
\text { en el medio } \\
\text { ambiente del } 1 \text { al } \\
5\end{array}$ & $\begin{array}{l}\text { Escala ordinal del } \\
1 \text { al } 5 \text {, donde } 1 \text { es } \\
\text { nada y } 5 \text { es } \\
\text { mucho. }\end{array}$ \\
\hline Género & $\begin{array}{l}\text { Variable } \\
\text { independiente } \\
\text { Especie humana }\end{array}$ & $\begin{array}{l}\text { ESIA Unidad } \\
\text { Tecamachalco } \\
\text { del IPN }\end{array}$ & $\begin{array}{l}\text { Hombre } \\
\text { Mujer }\end{array}$ & $\begin{array}{l}\text { Escala excluyente } \\
\text { y respuesta } \\
\text { directa } \\
\text { descriptiva- } \\
\text { nominal: } \\
\text { - H } \\
\text { - } \mathrm{M}\end{array}$ \\
\hline $\begin{array}{l}\text { Nivel } \\
\text { Académico }\end{array}$ & $\begin{array}{l}\text { Variable } \\
\text { independiente } \\
\text { Semestre académico } \\
\text { cursado por el } \\
\text { encuestado }\end{array}$ & $\begin{array}{l}\text { ESIA Unidad } \\
\text { Tecamachalco } \\
\text { del IPN }\end{array}$ & $\begin{array}{l}\text { Del 3. } .^{\circ} \text { al } 11 .^{\circ} \\
\text { semestre }\end{array}$ & $\begin{array}{l}\text { Directa ordinal: } \\
\text { - Del } 3 .^{\circ} \text { al } \\
11 .^{\circ}\end{array}$ \\
\hline
\end{tabular}

Fuente: Elaboración propia 


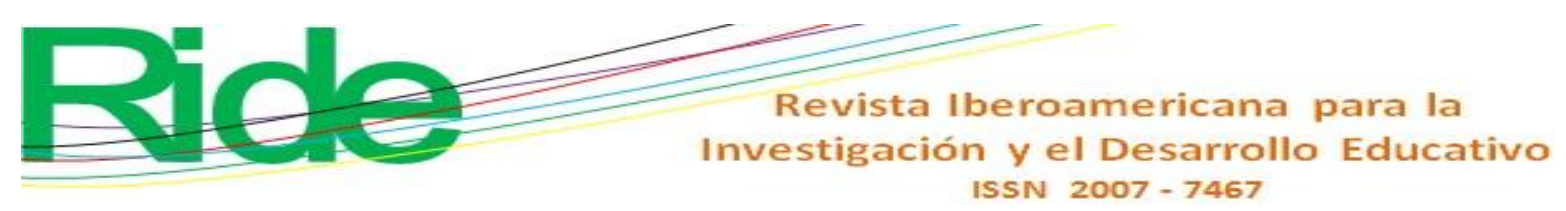

\section{Resultados}

De manera general, en cuanto a conocimientos de concepto de sustentabilidad, $96 \%$ manifestó estar familiarizado con el tema, lo cual, aparentemente, facilitó el estudio. Se les preguntó, además, sobre el conocimiento del origen del concepto y únicamente $54 \%$ fue positivo.

Asimismo, 69 \% resolvió que es muy importante relacionar la temática con la educación. Algo muy preocupante fue la respuesta manifestada hacia el gran desconocimiento (72\%) en relación con los programas de promoción y cuidado del medio ambiente de su unidad académica. Aquí, desafortunadamente, se infiere que los canales de comunicación o son insuficientes o inadecuados, pues los alumnos los ignoran.

Otra cuestión preocupante fue que pocos están enterados de la problemática vigente del deterioro ambiental: solo $45 \%$ refiere conocimiento al respecto.

Para realizar el análisis, tanto descriptivo como inferencial y utilizando el software SPSS, se tomó como referencia el modelo diseñado para ver la relación de las variables. En la tabla 2 se observa la población total, dividida por sexo.

Tabla 2. Datos de la población por sexo

\begin{tabular}{|l|l|r|r|r|r|}
\hline \multicolumn{2}{|c|}{} & Frecuencia & Porcentaje & $\begin{array}{l}\text { Porcentaje } \\
\text { válido }\end{array}$ & $\begin{array}{c}\text { Porcentaje } \\
\text { acumulado }\end{array}$ \\
\hline \multirow{4}{*}{ Válido } & Hombre & 88 & 50.9 & 50.9 & 50.9 \\
\cline { 2 - 6 } & Mujer & 85 & 49.1 & 49.1 & 100.0 \\
\cline { 2 - 6 } & Total & 173 & 100.0 & 100.0 & \\
\hline
\end{tabular}

Fuente: Elaboración propia

En la tabla 3, mientras tanto, se presenta el resultado referente al conocimiento del impacto negativo de sus hábitos cotidianos al cambio climático (vale la pena recordar: 1 es nada y 5 es mucho). Desafortunadamente, se aprecia que aún hay un grado de inconsciencia respecto a la problemática y sus consecuencias, ya que $38 \%$ señala que sus hábitos cotidianos impactan algo de manera negativa y $30 \%$ considera que impactan altamente. También se aprecia cómo la mujer es más sensible en el cuidado del medio ambiente, a pesar de que la población en hombres encuestados es mayor por las condiciones de la escuela. 


\section{Revista Iberoamericana para la Investigación y el Desarrollo Educativo ISSN $2007-7467$}

Tabla 3. Resultado de tabla cruzada entre variables

\begin{tabular}{|c|c|c|c|c|c|}
\hline \multirow{2}{*}{\multicolumn{3}{|c|}{ Variables }} & \multicolumn{2}{|c|}{ Sexo } & \multirow[b]{2}{*}{ Total } \\
\hline & & & Hombre & Mujer & \\
\hline \multirow[t]{15}{*}{ imp-neg } & \multirow[t]{3}{*}{ Nada } & Recuento & 1 & 2 & 3 \\
\hline & & Recuento esperado & 1.5 & 1.5 & 3.0 \\
\hline & & $\%$ dentro de Sexo & $1.1 \%$ & $2.4 \%$ & $1.7 \%$ \\
\hline & \multirow[t]{3}{*}{ Casi nada } & Recuento & 30 & 23 & 53 \\
\hline & & Recuento esperado & 27.0 & 26.0 & 53.0 \\
\hline & & $\%$ dentro de Sexo & $34.1 \%$ & $27.1 \%$ & $30.6 \%$ \\
\hline & \multirow[t]{3}{*}{ Algo } & Recuento & 27 & 38 & 65 \\
\hline & & Recuento esperado & 33.1 & 31.9 & 65.0 \\
\hline & & $\%$ dentro de Sexo & $30.7 \%$ & $44.7 \%$ & $37.6 \%$ \\
\hline & \multirow[b]{3}{*}{ Considerablemente } & Recuento & 18 & 12 & 30 \\
\hline & & Recuento esperado & 15.3 & 14.7 & 30.0 \\
\hline & & $\%$ dentro de Sexo & $20.5 \%$ & $14.1 \%$ & $17.3 \%$ \\
\hline & \multirow[t]{3}{*}{ Mucho } & Recuento & 12 & 10 & 22 \\
\hline & & Recuento esperado & 11.2 & 10.8 & 22.0 \\
\hline & & $\%$ dentro de Sexo & $13.6 \%$ & $11.8 \%$ & $12.7 \%$ \\
\hline \multirow{3}{*}{\multicolumn{2}{|c|}{ Total }} & Recuento & 88 & 85 & 173 \\
\hline & & Recuento esperado & 88.0 & 85.0 & 173.0 \\
\hline & & $\%$ dentro de Sexo & $100 \%$ & $100 \%$ & $100 \%$ \\
\hline
\end{tabular}

Fuente: Elaboración propia

En la tabla 4 se aprecia el análisis de la prueba ji al cuadrado: el resultado obtenido es mayor que 0.05. En este apartado, se acepta la H0, que refiere que son independientes, esto es, que la sensibilización (la respuesta) no depende del género. 


\section{Revista Iberoamericana para la Investigación y el Desarrollo Educativo ISSN 2007 - 7467}

Tabla 4. Pruebas de ji al cuadrado

\begin{tabular}{|l|r|r|r|}
\hline & \multicolumn{1}{|c|}{ Valor } & \multicolumn{1}{|c|}{ gl } & \multicolumn{1}{|c|}{$\begin{array}{c}\text { Significación } \\
\text { asintótica } \\
\text { (bilateral) }\end{array}$} \\
\hline Ji al cuadrado de Pearson & $4.451^{\mathrm{a}}$ & 4 & .348 \\
\hline Razón de verosimilitud & 4.476 & 4 & .345 \\
\hline $\begin{array}{l}\text { Asociación lineal por } \\
\text { lineal }\end{array}$ & .123 & 1 & .726 \\
\hline Núm. de casos válidos & 173 & & \\
\hline
\end{tabular}

${ }^{a}$ Dos casillas (20\%) han esperado un recuento menor que cinco. El recuento mínimo esperado es 1.47 .

Fuente: Elaboración propia

Teniendo en cuenta esto, se consideró necesario incluir una tercera variable, Nivel Académico, y especificar el semestre de estudio en curso del participante.

En la tabla 5 se muestra la distribución por semestre.

Tabla 5. Semestre en curso

\begin{tabular}{|l|l|r|r|r|r|}
\hline \multicolumn{2}{|c|}{} & Frecuencia & Porcentaje & Porcentaje válido & Porcentaje acumulado \\
\hline Válido & 3 & 7 & 4.0 & 4.0 & 4.0 \\
\cline { 2 - 6 } & 5 & 5 & 2.9 & 2.9 & 6.9 \\
\cline { 2 - 6 } & 6 & 10 & 5.8 & 5.8 & 12.7 \\
\cline { 2 - 6 } & 7 & 57 & 32.9 & 32.9 & 45.7 \\
\cline { 2 - 6 } & 8 & 26 & 15.0 & 15.0 & 60.7 \\
\cline { 2 - 6 } & 9 & 58 & 33.5 & 33.5 & 94.2 \\
\cline { 2 - 6 } & 10 & 8 & 4.6 & 4.6 & 98.8 \\
\cline { 2 - 6 } & 11 & 2 & 1.2 & 1.2 & 100.0 \\
\cline { 2 - 6 } & Total & 173 & 100.0 & 100.0 & \\
\hline
\end{tabular}

Fuente: Elaboración propia

Observando de manera distributiva, en la figura 2 se puede apreciar que sí existe una dependencia, en donde la mujer refiere una mayor sensibilización del impacto negativo que pueden tener sus actividades cotidianas hacia el medio ambiente. 


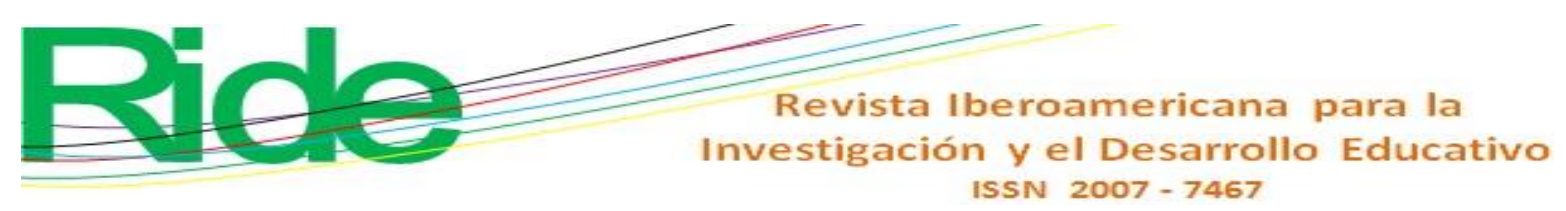

Figura 2. Correlación entre género y sensibilización ambiental

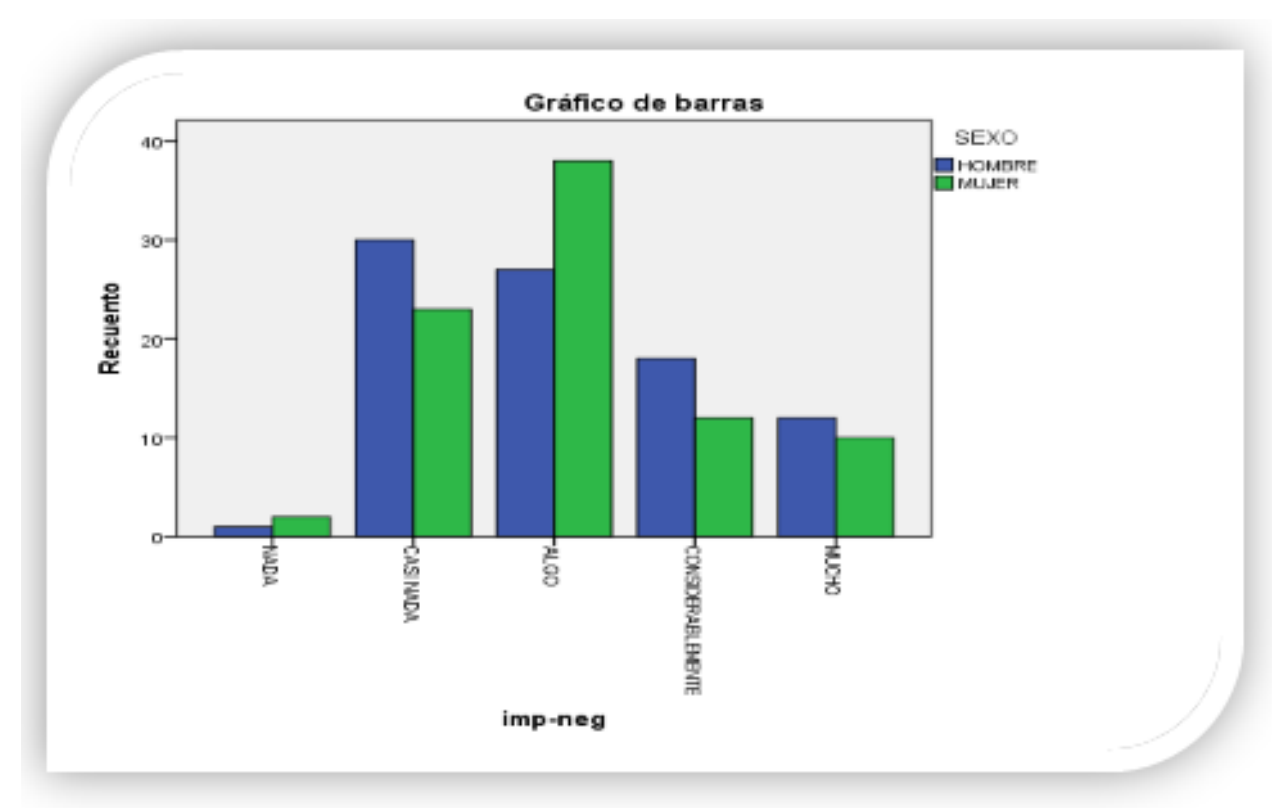

Fuente: Elaboración propia

Se realizó nuevamente la prueba con las tres variables y, enfatizando en el noveno semestre, se visualiza una dependencia directa entre la sensibilización ambiental y el género femenino. En la tabla 6 se visualiza que sí existe dependencia de las variables. El resultado es menor que 0.05, es decir, se rechaza la H0 (las variables son independientes) y se acepta la H1: las variables son dependientes. 


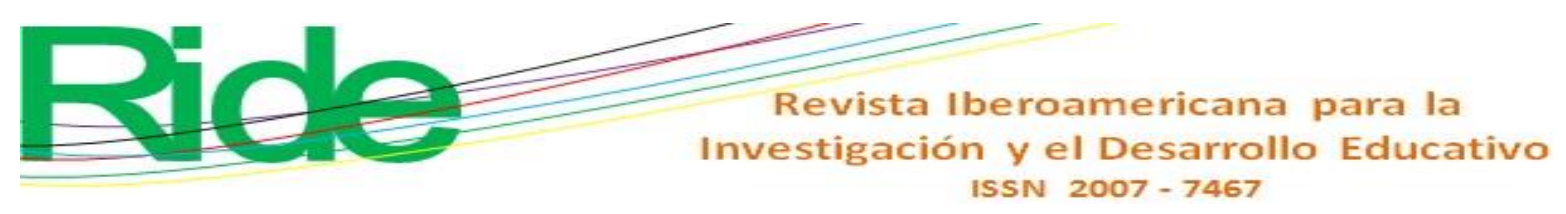

Tabla 6. Pruebas de ji al cuadrado

\begin{tabular}{|c|c|c|c|c|c|c|}
\hline \multicolumn{2}{|c|}{ Semestre } & \multirow{2}{*}{$\begin{array}{l}\text { Valor } \\
0.467^{\mathrm{b}}\end{array}$} & \multirow{2}{*}{$\begin{array}{r}\text { gl } \\
\\
1\end{array}$} & \multirow{2}{*}{$\begin{array}{c}\begin{array}{c}\text { Significación } \\
\text { asintótica } \\
\text { (bilateral) }\end{array} \\
0.495\end{array}$} & \multirow{2}{*}{$\begin{array}{c}\text { Significación } \\
\text { exacta } \\
\text { (bilateral) }\end{array}$} & \multirow{2}{*}{$\begin{array}{c}\text { Significación } \\
\text { exacta } \\
\text { (unilateral) }\end{array}$} \\
\hline 3 & $\begin{array}{l}\text { Ji al cuadrado de } \\
\text { Pearson }\end{array}$ & & & & & \\
\hline & $\begin{array}{l}\text { Corrección de } \\
\text { continuidad }^{\mathrm{c}}\end{array}$ & 0.000 & 1 & 1.000 & & \\
\hline & $\begin{array}{l}\text { Razón de } \\
\text { verosimilitud }\end{array}$ & 0.738 & 1 & 0.390 & & \\
\hline & $\begin{array}{l}\text { Prueba exacta de } \\
\text { Fisher }\end{array}$ & & & & 1.000 & 0.714 \\
\hline & $\begin{array}{l}\text { Asociación lineal por } \\
\text { lineal }\end{array}$ & 0.400 & 1 & 0.527 & & \\
\hline & $\begin{array}{l}\text { Núm. de casos } \\
\text { válidos }\end{array}$ & 7 & & & & \\
\hline \multirow[t]{6}{*}{5} & $\begin{array}{l}\text { Ji al cuadrado de } \\
\text { Pearson }\end{array}$ & $1.875^{\mathrm{d}}$ & 1 & 0.171 & & \\
\hline & $\begin{array}{l}\text { Corrección de } \\
\text { continuidad }^{\mathrm{c}}\end{array}$ & 0.052 & 1 & 0.819 & & \\
\hline & $\begin{array}{l}\text { Razón de } \\
\text { verosimilitud }\end{array}$ & 2.231 & 1 & 0.135 & & \\
\hline & $\begin{array}{l}\text { Prueba exacta de } \\
\text { Fisher }\end{array}$ & & & & 0.400 & 0.400 \\
\hline & $\begin{array}{l}\text { Asociación lineal por } \\
\text { lineal }\end{array}$ & 1.500 & 1 & 0.221 & & \\
\hline & $\begin{array}{l}\text { Núm. de casos } \\
\text { válidos }\end{array}$ & 5 & & & & \\
\hline 6 & $\begin{array}{l}\text { Ji al cuadrado de } \\
\text { Pearson }\end{array}$ & $4.286^{\mathrm{e}}$ & 3 & 0.232 & & \\
\hline
\end{tabular}




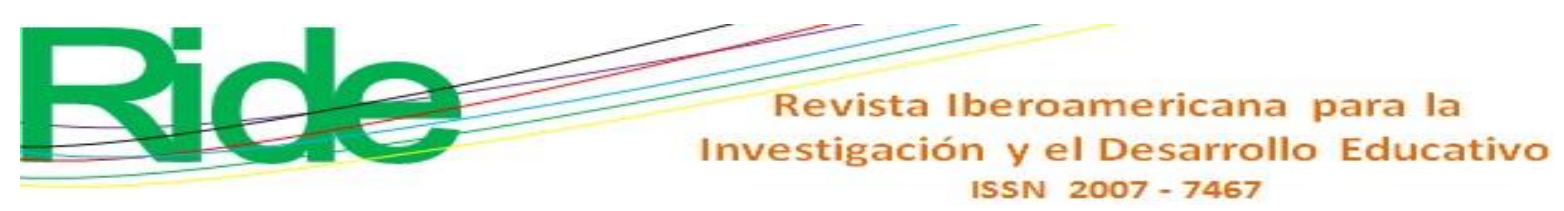

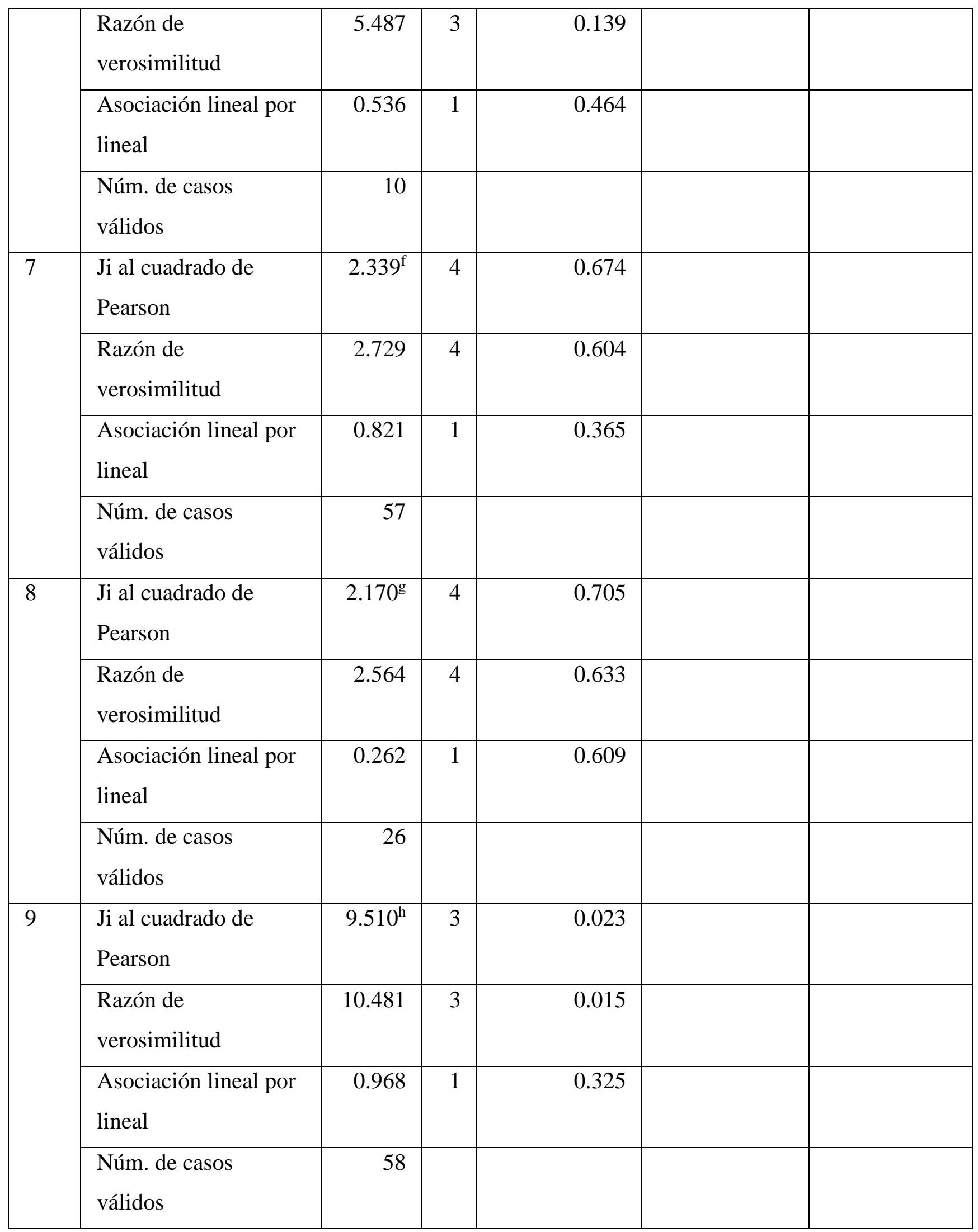




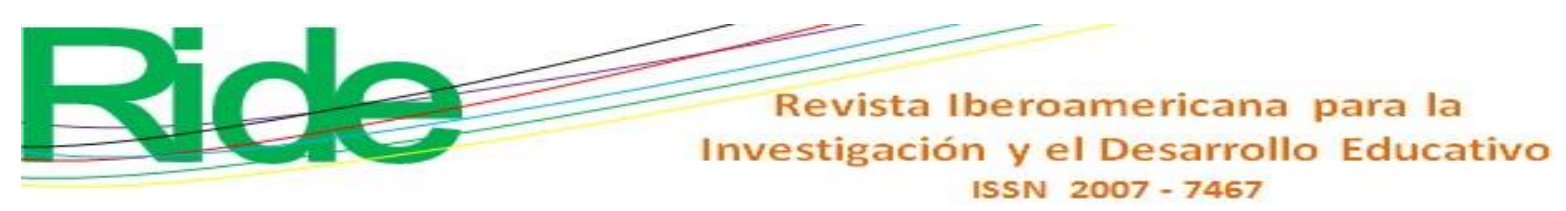

\begin{tabular}{|c|c|c|c|c|c|c|}
\hline \multirow[t]{4}{*}{10} & $\begin{array}{l}\text { Ji al cuadrado de } \\
\text { Pearson }\end{array}$ & $3.733^{\mathrm{i}}$ & 3 & 0.292 & & \\
\hline & $\begin{array}{l}\text { Razón de } \\
\text { verosimilitud }\end{array}$ & 5.040 & 3 & 0.169 & & \\
\hline & $\begin{array}{l}\text { Asociación lineal por } \\
\text { lineal }\end{array}$ & 0.614 & 1 & 0.433 & & \\
\hline & $\begin{array}{l}\text { Núm. de casos } \\
\text { válidos }\end{array}$ & 8 & & & & \\
\hline \multirow[t]{6}{*}{11} & $\begin{array}{l}\text { Ji al cuadrado de } \\
\text { Pearson }\end{array}$ & $2.000^{\mathrm{j}}$ & 1 & 0.157 & & \\
\hline & $\begin{array}{l}\text { Corrección de } \\
\text { continuidad }^{\mathrm{c}}\end{array}$ & 0.000 & 1 & 1.000 & & \\
\hline & $\begin{array}{l}\text { Razón de } \\
\text { verosimilitud }\end{array}$ & 2.773 & 1 & 0.096 & & \\
\hline & $\begin{array}{l}\text { Prueba exacta de } \\
\text { Fisher }\end{array}$ & & & & 1.000 & 0.500 \\
\hline & $\begin{array}{l}\text { Asociación lineal por } \\
\text { lineal }\end{array}$ & 1.000 & 1 & 0.317 & & \\
\hline & $\begin{array}{l}\text { Núm. de casos } \\
\text { válidos }\end{array}$ & 2 & & & & \\
\hline \multirow{4}{*}{ 苛 } & $\begin{array}{l}\text { Ji al cuadrado de } \\
\text { Pearson }\end{array}$ & $4.451^{\mathrm{a}}$ & 4 & 0.348 & & \\
\hline & $\begin{array}{l}\text { Razón de } \\
\text { verosimilitud }\end{array}$ & 4.476 & 4 & 0.345 & & \\
\hline & $\begin{array}{l}\text { Asociación lineal por } \\
\text { lineal }\end{array}$ & 0.123 & 1 & 0.726 & & \\
\hline & $\begin{array}{l}\text { Núm. de casos } \\
\text { válidos }\end{array}$ & 173 & & & & \\
\hline
\end{tabular}

${ }^{\text {a }}$ Dos casillas (20\%) han esperado un recuento menor que cinco. El recuento mínimo esperado es 1.47.

${ }^{\mathrm{b}}$ Cuatro casillas (100\%) han esperado un recuento menor que cinco. El recuento mínimo esperado es 0.29 .

${ }^{\mathrm{c}}$ Solo se ha calculado para una tabla $2 \times 2$.

${ }^{\mathrm{d}}$ Cuatro casillas (100\%) han esperado un recuento menor que cinco. El recuento mínimo esperado es 0.40 . 


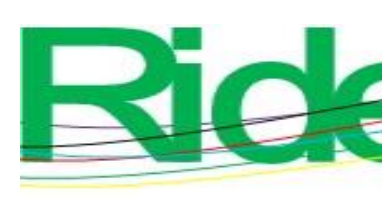

\section{Revista Iberoamericana para la Investigación y el Desarrollo Educativo ISSN $2007-7467$}

e Ocho casillas (100\%) han esperado un recuento menor que cinco. El recuento mínimo esperado es 0.30.

${ }^{\mathrm{f}}$ Tres casillas (30 \%) han esperado un recuento menor que cinco. El recuento mínimo esperado es 0.47.

g 10 casillas (100\%) han esperado un recuento menor que 5. El recuento mínimo esperado es 0.46.

h Tres casillas (37.5\%) han esperado un recuento menor que cinco. El recuento mínimo esperado es 2.33.

${ }^{\mathrm{i}}$ Ocho casillas $(100 \%)$ han esperado un recuento menor que cinco. El recuento mínimo esperado es 0.38.

${ }^{\mathrm{j}}$ Cuatro casillas $(100 \%$ ) han esperado un recuento menor que cinco. El recuento mínimo esperado es 0.50.

\section{Fuente: Elaboración propia}

Los resultados obtenidos indican (en específico en el noveno semestre) que sí existe una dependencia en la respuesta por sexo, en donde la mujer manifiesta una mayor sensibilidad en el cuidado del ambiente.

\section{Discusión}

Resulta contrastante analizar que, a pesar de ser una temática trabajada desde antaño (más de tres décadas), el involucramiento de la mujer en diferentes actividades humanas continúa manejándose a nivel teórico o discursivo.

En una primera instancia, los resultados arrojaron que no existe relación entre el género y la sensibilidad ambiental. Pudiera ser porque la población en donde se realizó el estudio es predominantemente masculina y están inscritos a una licenciatura del área de ingeniería. Sería conveniente, en un estudio posterior, incrementar el tamaño de la muestra o extender el estudio a otras escuelas o instituciones.

Paradójicamente, resulta una situación de poco involucramiento formal de la mujer en cuestiones de cuidado e impacto en el medio ambiente. Existen muchos discursos para mantener a hombres y mujeres en sus mundos aparentemente separados de ciencia, economía y defensa altamente valorados, con lo que se devalúa la reproducción social y el deber doméstico privado por el otro. Los discursos masculinistas dan forma al problema de manera que excluyen efectivamente a las mujeres (MacGregor, 2010).

Por otro lado, cabe señalar que el involucramiento más directo de la mujer en el cuidado del medio ambiente ha sido marginado, así como la inclusión en aspectos de toma de decisiones impactantes. En esta institución educativa, los encargos superiores los ocupan los varones, por tal motivo es conveniente trabajar en acciones que involucren a la mujer en liderar proyectos que impacten directamente en su entorno inmediato, como es su hogar y entorno social, ya que podría ser un motivo para generar ingresos familiares, tal y como se ha realizado desde los años 80 en Nepal, en donde se trabajaron proyectos para integrar a la mujer en aspectos específicos para 


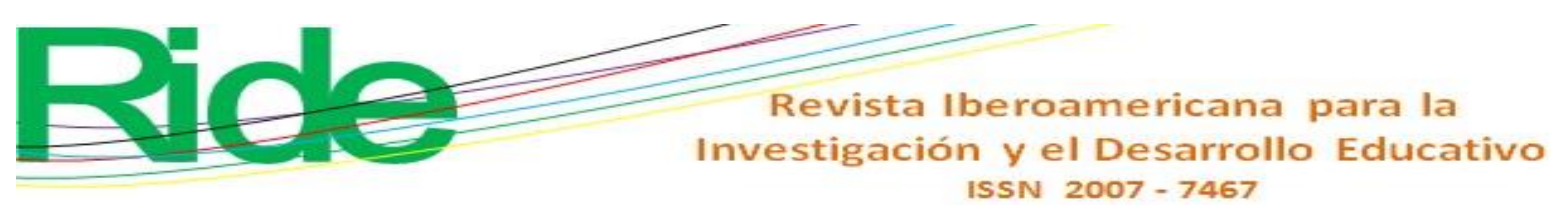

ayudarse a sí mismas y a otras mujeres a mejorar su situación económica (Molnar, 1987). Estos programas han manifestado que los tomadores de decisiones deben observar el hecho de que los proyectos de generación de ingresos para y por las mujeres son viables y tienen papeles importantes en el desarrollo regional (Molnar, 1987).

Existe poca información sobre la diferencia de cuál es el comportamiento por género sobre la responsabilidad que se tiene de cuidar el medio ambiente. En un artículo publicado por la Unesco, referente al papel que desempeñan sobre todo las mujeres en relación con indicadores hídricos, se manifiesta que es imposible medir los progresos hacia los objetivos del desarrollo sostenible (Seager, 2015). Desafortunadamente, el papel de la mujer en aspectos tan importantes como es el cambio climático resulta únicamente un tanto simbólico. Las mujeres representan físicamente los números mínimos, pero no necesariamente tienen una opinión decisiva (Sharma, 2013). Es por ello por lo que las mujeres surgieron como una fuerza, no solo en apoyo de una gestión ambiental adecuada, también en demandas de una mejor calidad de vida y una mayor equidad social (Rico, 1998).

La presente investigación permite visualizar de una manera objetiva el compromiso humano, para integrarse como una sociedad responsable y dirigirse a través de una educación integral y bajo principios axiológicos que conlleven a una cultura ambiental. La principal fortaleza es que permite abrir la puerta a estudios más amplios para fundamentar y reivindicar el papel y compromiso de la mujer como persona, con una capacidad de respuesta, trabajar de manera conjunta y equitativa con el varón, con la oportunidad de propiciar el bienestar.

\section{Conclusiones}

Es necesario resaltar que la temática abordada en esta investigación, la correlación del género y el nivel académico con la sensibilidad ambiental (conciencia del impacto ambiental negativo del individuo en su devenir), no es un tema muy estudiado, sobre todo en cuestiones de impacto; la mayoría de los estudios se quedan en un nivel de intención y formulación estratégica.

El objetivo general se cumplió al lograr señalar la importancia de la educación dirigida hacia la conformación de una cultura ambiental, partiendo de un enfoque de género y de nivel académico con su implicación y dirección hacia la generación de una sensibilidad ambiental, complementando lo anterior con las respuestas colaterales señaladas en el cuestionario y en las entrevistas (cuestionario semiestructurado). 


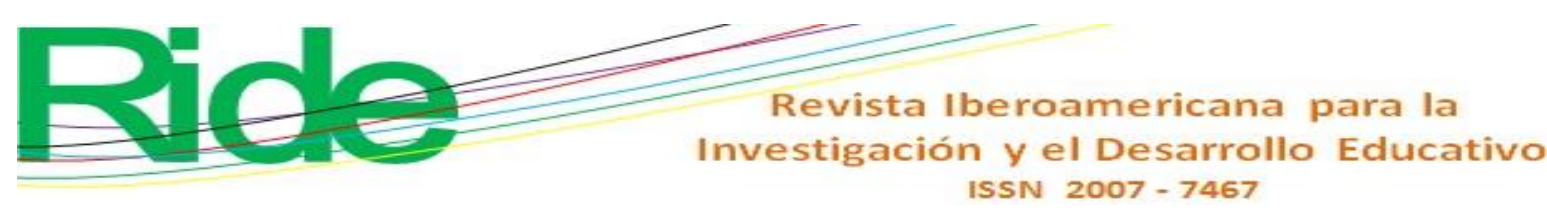

Se manifestó la tendencia femenina en cuanto a la conciencia de impactar en menor grado en el deterioro ambiental al aprobar la $\mathrm{H} 1$ en la parte cuantitativa, desde el punto de vista estadístico. Al final del estudio, se encontró que el género y el nivel académico influyen de manera directa en la respuesta sensible del individuo hacia el cuidado del medio ambiente, y que la mujer puede propiciar una educación ambiental desde el hogar para que el individuo la complemente en la escuela, y sobre todo, la pueda vivir y disfrutar en sociedad, lo que repercutirá en la construcción de una cultura ambiental.

Como fundamento se menciona los siguiente: resulta paradójico que los términos relacionados con el cambio climático y deterioro ambiental: casi el total de los encuestados los conocían y estaban familiarizados con ellos, sin embargo, únicamente $69 \%$ de los participantes consideró pertinente relacionarlos en el ámbito educativo. También resultó preocupante que $72 \%$ desconociera si la unidad académica en cuestión tenía programas específicos o promovía acciones en pro de la sustentabilidad. Desafortunadamente, se puede inferir que los canales de comunicación o son insuficientes o inadecuados. Algo también no menos preocupante fue que únicamente $45 \%$ están enterados de la problemática vigente del deterioro ambiental.

Por otro lado, es necesario seguir realizando investigaciones que midan el impacto humano en diferentes ambientes, y poner especial énfasis en el género, ya que, como dice la Unesco, no por decreto o buenas intenciones político-sociales el individuo va a cambiar de conducta y tener plena conciencia de nuestra corresponsabilidad axiológica y pragmática de manifestar una cultura sostenible que impacte de manera positiva en el medio ambiente para que sigamos disfrutando lo que nos proporciona la naturaleza, lo podamos preservar y garanticemos el gozo a futuras generaciones.

Para fortalecer este resultado, se recomienda continuar con un estudio que aplique el coeficiente phi de Pearson o la $V$ de Cramer (adecuando las tablas de contingencia) para una interpretación más específica y medir el nivel de la significancia de la correlación.

\section{Agradecimientos}

Instituto Politécnico Nacional, Proyectos: SIP-20171478 "Sustentabilidad en el consumo de energía de grandes edificios habitacionales" y SIP-20195789 “Administración de recursos materiales en hogares en estado de pobreza". 


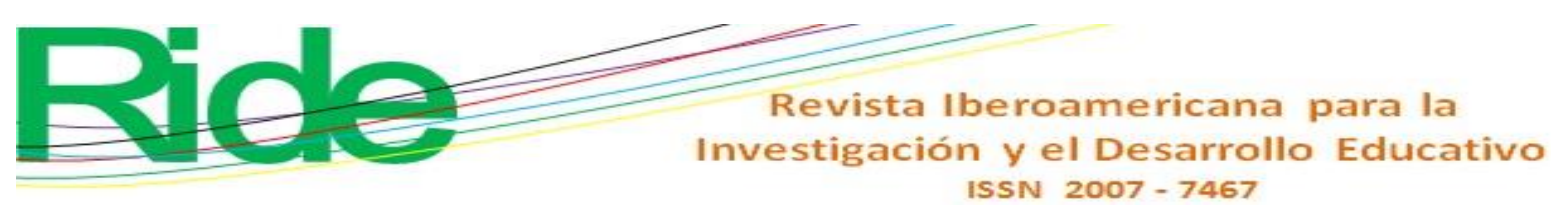

\section{Referencias}

Caride, J. y Meira, P. Á. (1998). Educación ambiental y desarrollo: la sustentabilidad y lo comunitario como alternativas. Pedagogía Social: Revista Interuniversitaria, (2), 7-30.

Dieleman, H. y Juárez, M. (2008). ¿Cómo se puede diseñar educación para la Sustentabilidad?. Revista Internacional de Contaminación Ambiental, 24(3). Recuperado de http://www.scielo.org.mx/scielo.php?script=sci_arttext\&pid=S018849992008000300004.

Escuela Superior de Ingeniería y Arquitectura (ESIA) Unidad Tecamachalco. (2019) Misión y Visión. México: Instituto Politécnico Nacional. Recuperado de https://www.esiatec.ipn.mx/conocenos/mision-vision.html.

Flogaitis, E. (1998). The contribution of environmental education in sustainability. In Scoullos, M. (ed.), Environment and Society: Education and Public Awareness for Sustainability. Greece, Athens: University of Athens.

Hernández, R., Fernández, C. y Baptista, P. (2004). Metodología de la Investigación. México: McGraw-Hill.

Instituto Politécnico Nacional [IPN]. (28 de mayo de 1982). Ley Orgánica del Instituto Politécnico Nacional. Diario Oficial de la Federación. Recuperado de http://www.diputados.gob.mx/LeyesBiblio/pdf/171.pdf.

KPMG en México. (2018). Desarrollo sostenible en México 2018. Ciudad de México, México: KPMG en México. Recuperado de http://www.pactomundial.org.mx/site/wpcontent/uploads/2018/03/KPMG-Estudio-Desarrollo-Sostenible-en-Mexico2018.pdf.

Levin, R. y Rubin, D. (2004). Estadística para administración y economía (7.a ed.). México: Pearson Educación.

MacGregor, S. (2010). Gender and climate change': from impacts to discourses. Journal of the Indian Ocean Region, 6(2).

Miranda, L. M. (2013). Cultura ambiental: un estudio desde las dimensiones de valor, creencias, actitudes y comportamientos ambientales. Producción + Limpia, 8(2), 94-105.

Molnar, A. (1987). Forest conservation in Nepal: Encouraging women's participation. Seeds, (10), $1-20$.

Organización de las Naciones Unidas [ONU]. (1987). Report of the World Commission on Environment and Development: Our Common Future. Organización de las Naciones Unidas. 


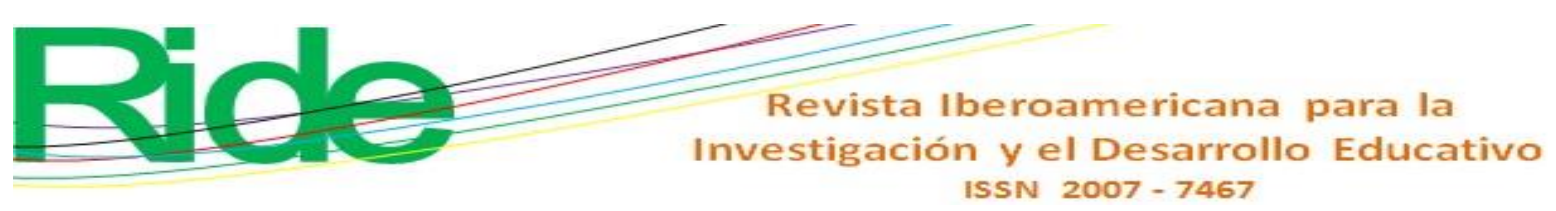

Organización de las Naciones Unidas para la Educación, la Ciencia y la Cultura [Unesco]. (2005).

Decenio de las Naciones Unidas de la Educación para el Desarrollo Sostenible (20052014): el Decenio en pocas palabras. París, Francia: Organización de las Naciones Unidas para la Educación, la Ciencia y la Cultura.

Organización de las Naciones Unidas para la Educación, la Ciencia y la Cultura [Unesco]. (2006a).

Decenio de las Naciones Unidas de la Educación para el Desarrollo Sostenible (20052014): plan de aplicación internacional. París, Francia: Organización de las Naciones Unidas para la Educación, la Ciencia y la Cultura.

Organización de las Naciones Unidas para la Educación, la Ciencia y la Cultura [Unesco]. (2006b).

Sustainable Development Data Digest: Laying the Foundation to Measure Sustainable

Development Goal 4. Paris, France: Organización de las Naciones Unidas para la Educación, la Ciencia y la Cultura.

Organización de las Naciones Unidas para la Educación, la Ciencia y la Cultura [Unesco]. (2010).

Sandwatch: adapting to climate change and educating for sustainable development. Paris, France: Organización de las Naciones Unidas para la Educación, la Ciencia y la Cultura.

Organización de las Naciones Unidas para la Educación, la Ciencia y la Cultura [Unesco]. (2014).

Gender equality and sustainable development. United States: Organización de las Naciones Unidas para la Educación, la Ciencia y la Cultura.

Organización de las Naciones Unidas para la Educación, la Ciencia y la Cultura [Unesco]. (2018).

Issues and trends in Education for Sustainable Development. France, Paris: Organización de las Naciones Unidas para la Educación, la Ciencia y la Cultura.

Organización para la Cooperación y el Desarrollo Económicos [OCDE]. (2019). El futuro de la educación superior en méxico: promoviendo calidad y equidad. Organización para la Cooperación y el Desarrollo Económicos. Recuperado de https://www.oecd.org/centrodemexico/medios/el_futuro_de_la_educacion_en_mexico.pd f.

Padua, J. (2016). Técnicas de investigación aplicadas a las ciencias sociales. México: Colegio de México / FCE.

Presidencia de la República. (2019). Plan Nacional de Desarrollo-2019-2024. México: Presidencia de la República. Recuperado de https://lopezobrador.org.mx/wpcontent/uploads/2019/05/PLAN-NACIONAL-DE-DESARROLLO-2019-2024.pdf. 


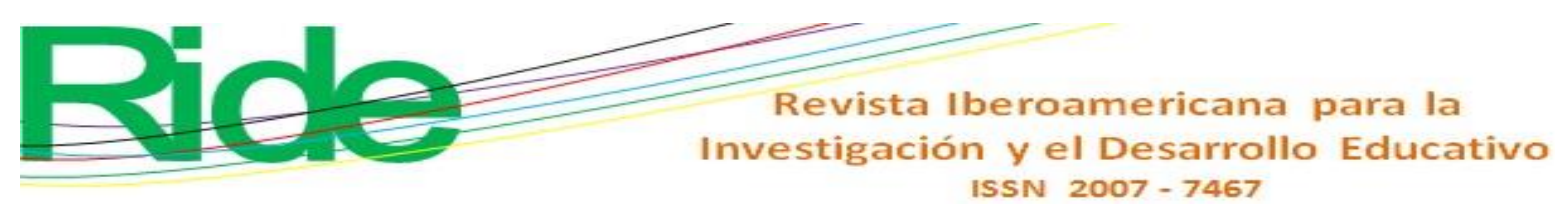

Programa de las Naciones Unidas para el Medio Ambiente [UNEP]. (2016). Global Gender and Environment Outlook. Nairobi, Kenya: Programa de las Naciones Unidas para el Medio Ambiente.Rico, M. N. (1998). Gender, the environment and the sustainability of development. Santiago, Chile: United Nations.

Sandoval, M. (2012). Comportamiento sustentable y educación ambiental: una visión desde las prácticas culturales. Revista Latinoamericana de Psicología, 44(1), 181-196.

Santín del Río, L. (2011). En el camino de la democracia partidaria. Perspectivas y paradojas. En Ackerman J. M. (coord.), Elecciones 2012: En busca de equidad y legalidad (pp. 303-310). Ciudad de México, México: Universidad Nacional Autónoma de México.

Seager, J. (2015). Indicadores desglosados por sexo para la presentación de informes, evaluación y seguimiento de recursos hídricos. París, Francia: Organización de las Naciones Unidas para la Educación, la Ciencia y la Cultura.

Secretaría de Gobernación. (2016). Constitución Política de los Estados Unidos Mexicanos. Ciudad de México, México: Secretaría de Gobernación.

Secretaría de Medio Ambiente y Recursos Naturales [Semarnat]. (2019). México, larga experiencia en educación y cultura ambiental. México: Secretaría de Medio Ambiente y Recursos Naturales. Recuperado de https://www.gob.mx/semarnat/articulos/mexico-largaexperiencia-en-educacion-y-cultura-ambiental?idiom=es.

Sharma, K. (2013). Gender and green governance: the political economy of women's presence within and beyond community forestry. Journal of Human Development and Capabilities, 14(2), 314-316. 